\title{
DETECTOR DE OBSTÁCULOS PARA DEFICIENTES VISUAIS COM ARDUINO
}

\author{
Artur Auresco Damasio' \\ Gabriel Henrique Faria Gonçalez ${ }^{2}$ \\ Pedro André Etelvino dos Santos ${ }^{3}$ \\ Rafael Machado do Prado 4 \\ Robson Lopes Junior ${ }^{5}$ \\ Vinícius Yuji Guima ${ }^{6}$
}

Amanda Sant' Anna Sciammarella Montecin ${ }^{7}$

Resumo: A tecnologia nos tempos atuais deve ser inclusiva e acessível, além de ser uma maneira de melhorar a qualidade de vida, inclusive dos deficientes visuais. Pensado nisso desenvolveu-se um protótipo de luva ultrassônica que tem por objetivo melhorar a segurança durante a locomoção dos que não possuem o sentido da visão. Com isso, pôde-se propor uma alternativa com baixo custo que usa apenas um Arduino associado a um sensor que emite pulsos ultrassônicos, pelos quais é possível determinar a distância, que por meio do motor vibracall é convertido em vibrações que aumentam de intensidade conforme se aproxima de algum objeto.

Palavras-chave: Deficiente visual; Luva; Econômico; Arduino; Ultrassônico.

\footnotetext{
1 Programa Pré Universitário Pré Exatas/Colégio Embraer Juarez Wanderley, Brasil. E-mail: art.damasio13@gmail.com.

2 Programa Pré Universitário Pré Exatas/Colégio Embraer Juarez Wanderley, Brasil. E-mail: goncalezgabriel@gmail.com.

3 Programa Pré Universitário Pré Exatas/Colégio Embraer Juarez Wanderley. Brasil. E-mail: pedro7900@hotmail.com.br.

${ }^{4}$ Programa Pré Universitário Pré Exatas/Colégio Embraer Juarez Wanderley. Brasil. E-mail: rafael.m.prado@hotmail.com.

5 Programa Pré Universitário Pré Exatas/Colégio Embraer Juarez Wanderley. E-mail: robsonLopesJunior@outlook.com.

${ }^{6}$ Programa Pré Universitário Pré Exatas/Colégio Embraer Juarez Wanderley. E-mail: viyugui@gmail.com.

${ }^{7}$ Colégio Embraer Juarez Wanderley, Brasil. E-mail: mandy_santanna@hotmail.com.
} 